\title{
Fingerprint Sensor Circuit and Sensor Cell Isolation Scheme for Pseudo-Direct Signaling
}

\author{
Hyeopgoo Yeo \\ Division of Information \& Telecommunications, Hanshin University, Osan-Si, \\ Korea \\ hgyeo@hs.ac.kr
}

\begin{abstract}
This paper proposes a fingerprint sensor cell circuit structure and a sensor cell isolation schemes for a pseudo-direct scheme. The proposed fingerprint sensor cell is used as both sensing plate detecting transmit(Tx) signal and Tx electrode transmitting Tx signal. The evaluation sensor cells use the sensor plates as sensing electrodes otherwise the sensor plates are used as Tx electrodes, which enables the fingerprint sensor to form a enough capacitance between the Tx electrodes and a finger. Moreover, it can reduce the chip size by removing the Tx electrodes that occupies a large area of the chip. The proposed fingerprint sensor circuit were designed with an active output voltage feedback

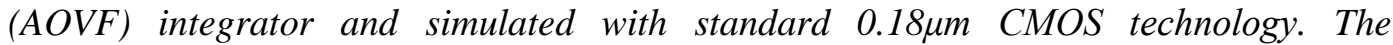
simulation results verified that the proposed fingerprint sensor using pseudo-direct scheme will work well even in thick film without an external electrode that drives a sensor signal to a finger. Therefore, the fingerprint sensor with the proposed sensor cell circuit and the sensor cell isolation scheme can be effectively used as a personal authentication method especially in mobile applications.
\end{abstract}

Keywords: fingerprint sensor, pseudo-direct scheme, sensor cell isolation, AOVF integrator, signal integration scheme

\section{Introduction}

Demand for fingerprint sensors is growing rapidly in mobile system as a means of biometric recognition[1, 2]. In particular, fingerprint sensor is used as means of personal identification method in mobile system. A capacitive fingerprint sensor is the best type of fingerprint sensor for mobile systems because it is cheap and small in size. The capacitive sensor senses the change in capacitance formed between a sensor plate and a finger. The capacitance of the fingerprint sensor decreases as the thickness of the passivation film between the sensor plate and a finger increases. To improve the performance of fingerprint sensing, a method of directly transmitting a signal to a finger using a bezel has been proposed[3, 4]. Placing a bezel not only restricts the fingerprint sensor module design but also increases the cost especially in mobile system. Therefore, the demand for a bezel-less fingerprint sensor in mobile systems has increased. However, a bezel-less fingerprint sensor has a relatively lower performance compared to the fingerprint sensor using bezel. A fingerprint sensor using pseudo-direct scheme which does not use bezel has been introduced[5]. Large area of $\mathrm{Tx}$ electrode is required to form enough capacitances between a Tx electrode and a finger for sufficient sensor signal transmission to a finger. To form enough capacitance, Tx electrode in a fingerprint sensor surrounds the fingerprint sensor cell array as shown in Figure 1(b)[5]. However, this large area of Tx electrode located outside of the sensor cell array increases chip size significantly.

This paper proposes a fingerprint sensor cell circuit structure and sensor cell isolation scheme for pseudo-direct signal transmissions. The proposed fingerprint sensor cell was 
modeled and simulated using $0.18 \mu \mathrm{m}$ CMOS technology. The operating principle of the proposed fingerprint sensor using pseudo-direct signal transmission was explained and simulation results were presented in this paper.

\section{Fingerprint Sensor Circuit with Pseudo-direct Scheme}

Figure 1(a) shows the fingerprint sensor's circuit structure with pseudo-direct scheme proposed in [5]. The equivalent circuit of a finger are simply modeled as a series connected resistor, $\mathrm{R}_{S}$, and shunt resistor between a finger and an external ground, $\mathrm{R}_{\mathrm{P}}$, and $\mathrm{C}_{\text {finger }}$ formed between a finger and a sensor plate of a sensor cell as shown in Figure 1(b). Note that, $\mathrm{C}_{\text {finger }}$ can be either $\mathrm{C}_{\text {ridge }}$ or $\mathrm{C}_{\text {valley }}$. A capacitor formed between a finger and a Tx electrode is modeled as $\mathrm{C}_{\mathrm{Tx}}$. Tx electrode surrounding chip the fingerprint sensor cell array can be made of a top metal and as shown in Figure 1(b). $\mathrm{C}_{\mathrm{Tx}}$ is a modeled capacitor formed between the Tx electrode and a finger. $R_{P}$ is a shunt resistor at the point of $C_{T x}$ to an external ground through the finger. $\mathrm{R}_{\mathrm{S}}$ and $\mathrm{C}_{\text {finger }}$ are a series-connected resistor and a capacitor as shown in Figure 1(a). Figure 1(c) shows the vertical layout view of the two adjacent fingerprint sensor cells for pseudo-direct scheme including Tx electrode. Obviously, the area of Tx electrode should be large to form enough capacitance between Tx electrode and finger, $\mathrm{C}_{\mathrm{Tx}}$. The capacitance between the fingerprint sensor array and Tx electrode, which is denoted as $\mathrm{C}_{\text {gap }}$, should be small to minimize the direct inflows of the electric fields to the sensor cell generated from the Tx electrode. This causes an increases in chip size of the fingerprint sensor with pseudo-direct scheme.

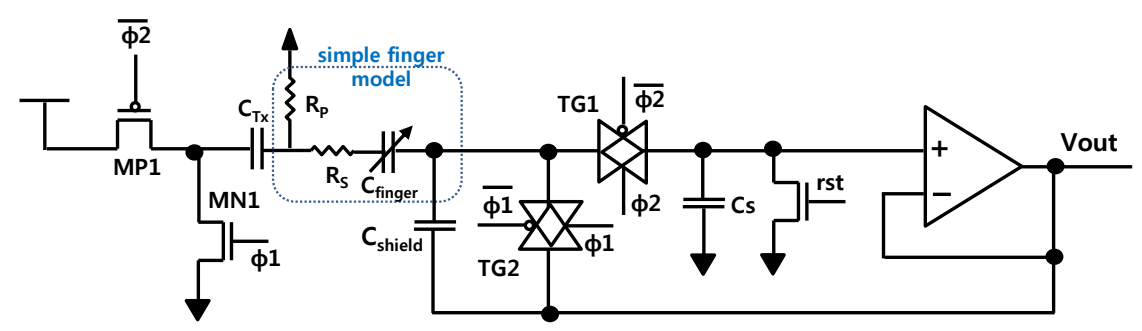

(a)

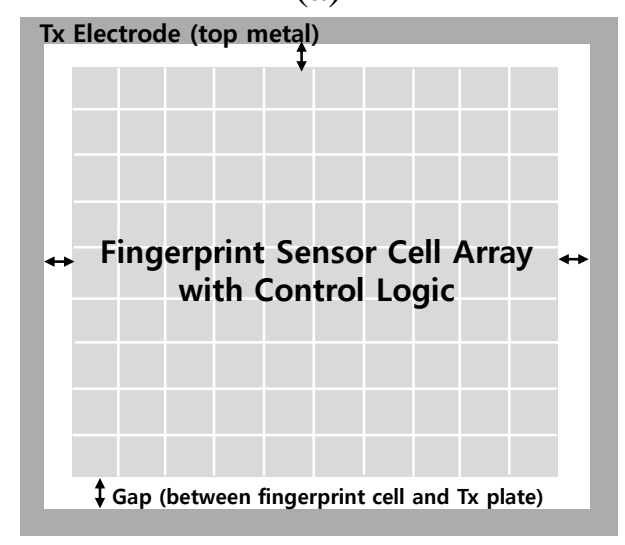

(b) 


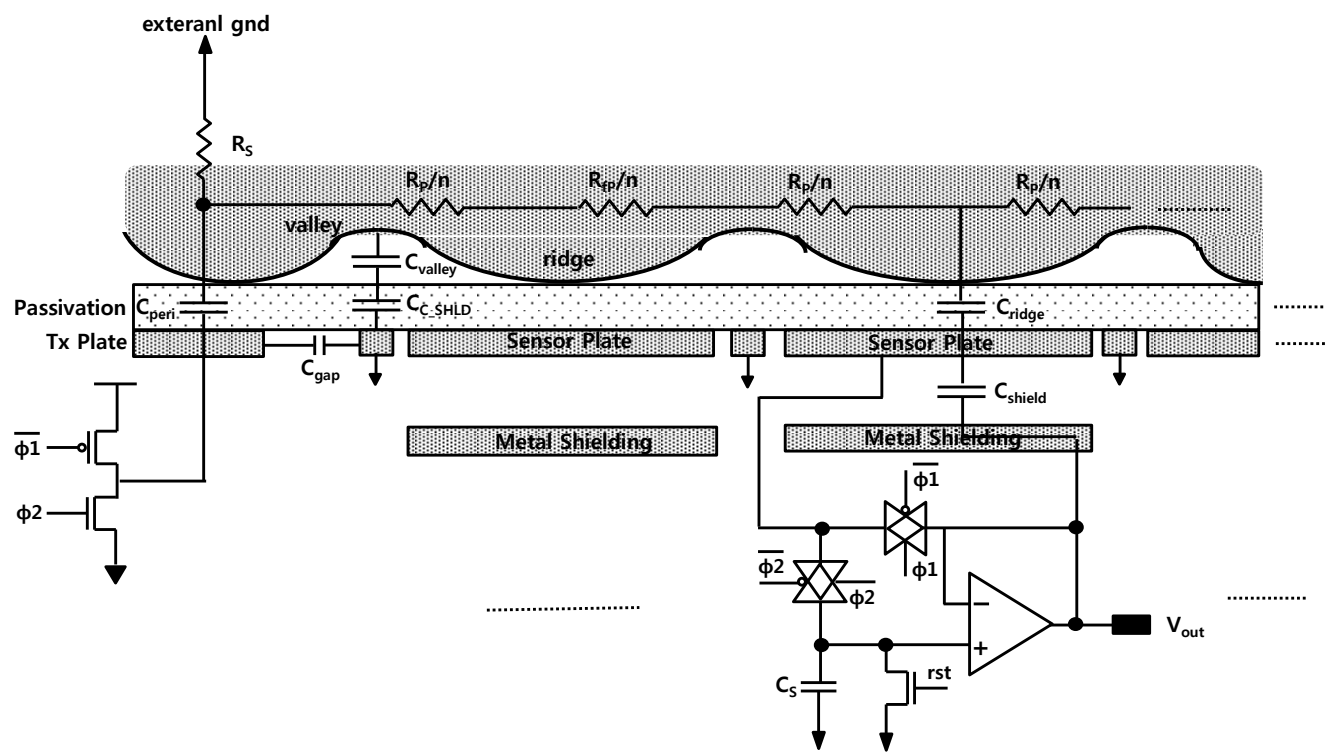

(c)

\section{Figure 1. (a) The Fingerprint Sensor Cell Circuit with Pseudo-direct Scheme (b) Vertical Layout Structure of the Fingerprint Sensor Cell Unit Applied in MOS Technology (c) Top Layout View of the Fingerprint Sensor Array with Pseudo-direct Scheme}

\section{Proposed Reusing Fingerprint Sensor Cell Circuit and Structure}

This paper proposes a fingerprint sensor cell circuit and structure reusing sensor cells and their control scheme for the pseudo-direct scheme. Figure 2(a) shows the proposed a fingerprint sensor cell circuit. Not only the sensor cell receives a sensor signal through the $\mathrm{C}_{\text {finger }}$ but also it is designed to send Tx signal through the $\mathrm{C}_{\text {finger }}$. A CMOS analog switch is added for passing Tx signal to $\mathrm{C}_{\text {finger }}$ and a NMOS switch for isolating a sensor cell by grounding the $\mathrm{C}_{\text {finger. }}$. Because a fingerprint sensor evaluates a sensor cell one by one or a few sensor cells, most of the remaining sensor cells do nothing. Therefore, the remaining sensor cell can be reused as a Tx electrode transmitting the SDRV signal by enabling the CMOS switch with EN as shown in Figure 2(a). In this way, the proposed fingerprint sensor can reuse a sensor cell as a Tx electrode not only a sensor cell sensing the signal. Therefore, many Tx sensor cells exist inside the sensor cell array. As a result, many Tx electrodes form enough capacitance between the Tx electrodes and a finger effectively without increasing the additional chip area. In addition, an isolation sensor cell is designed for blocking the direct inflows of the Tx signal into an evaluation sensor cell. NMOS switch with ENISOL selects an isolation sensor cell by grounding the $\mathrm{C}_{\mathrm{Tx}}$ while the other unselected sensor cells are used as a Tx electrode. Three kinds of sensor cells exist in the proposed fingerprint sensor by controlling the switches in the sensor cell. Those are an evaluation sensor cell, a Tx sensor cell, and an isolation sensor cell. The isolation sensor cells are located between an evaluation sensor cell and Tx sensor cells in order to block the inflows of the Tx signals generated from the adjacent Tx sensor cells. Figure 2(b) shows the vertical layout structure of the proposed fingerprint sensor cell. A CMOS switch and a NMOS switch is added to a sensor plate so as to it can be used as a Tx electrode signal or an isolation electrode. As shown in Figure 2(b), Tx signal reaches the sensor plate through the CMOS switch for Tx sensor cell and the sensor plate is tied to ground via the NMOS for isolation sensor cell. Of course, an evaluation sensor cell disables both the NMOS switch and the CMOS switch for normal evaluation operations. 


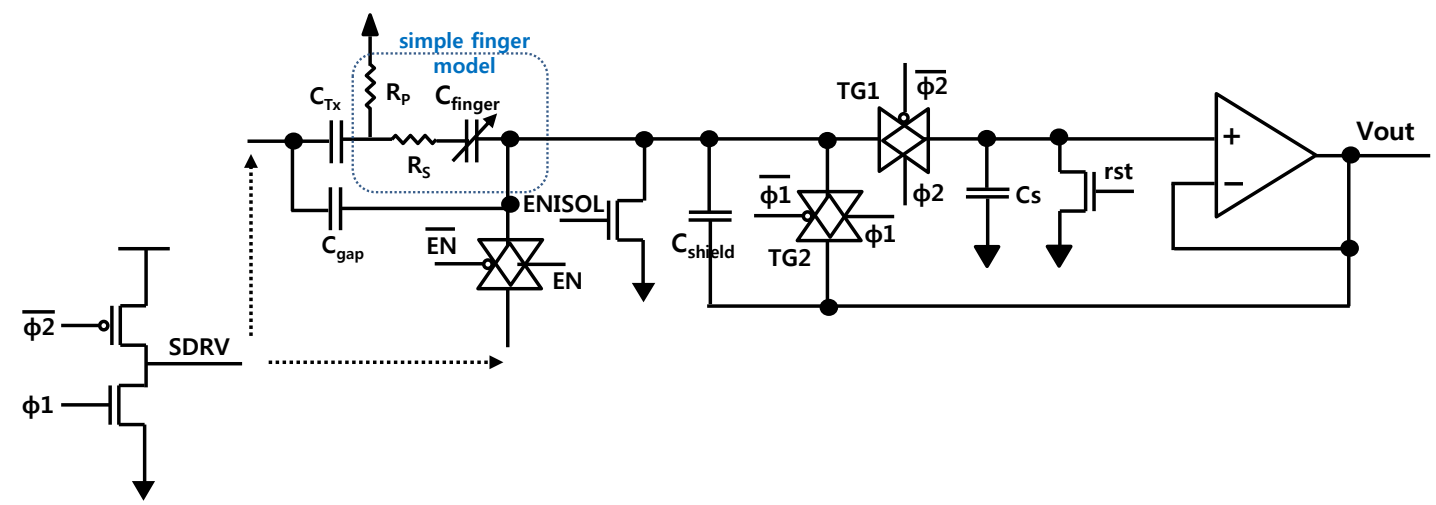

(a)

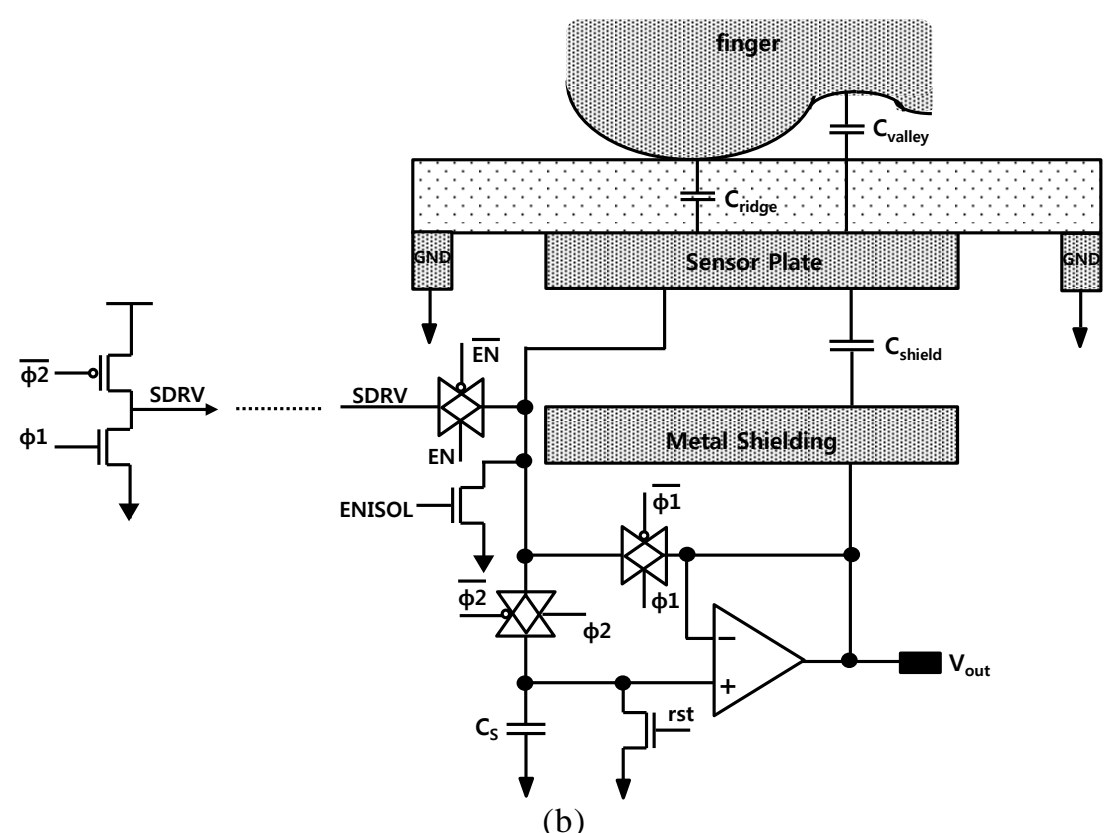

Figure 2. (a) The Proposed Reusing Fingerprint Sensor Cell Circuit for Pseudo-direct Scheme (b) Vertical Layout Structure of the Proposed Fingerprint Sensor Cell Unit Applied in MOS Technology

Figure 3(a) shows the top layout view of the part of proposed fingerprint sensor cell array for a pseudo-direct scheme which includes an evaluation sensor cell, Tx sensor cells. The center sensor cell is an evaluation cell while the other cells are Tx sensor cells. As mentioned, it is very important to minimize $C_{\text {gap }}$ so that it blocks the direct inflows of electric fields generated from Tx electrodes, which helps correct evaluation process for an evaluation cell. However, Tx sensor cells are adjacent to the evaluation sensor cell as shown in Figure 3(a), the electric fields from the Tx electrodes come into from everywhere which causes invalid evaluation. Figure 3(b) shows the electric fields for the evaluation sensor cell and the Tx sensor cell roughly. The Tx electric fields should be absorbed by a finger and then retransmitted to the evaluation sensor cell for the proper evaluation. Part of electric fields generated from Tx sensor cells could reach the evaluation sensor cell directly because the gap between the evaluation sensor cell and $\mathrm{Tx}$ sensor cells are very close. If the passivation film becomes thicker, the direct inflows of electric fields generated from Tx sensor cells will more affect the sensor cell evaluation process because the electric fields to a finger becomes weak while the direct inflows of electric fields remains almost the same. 


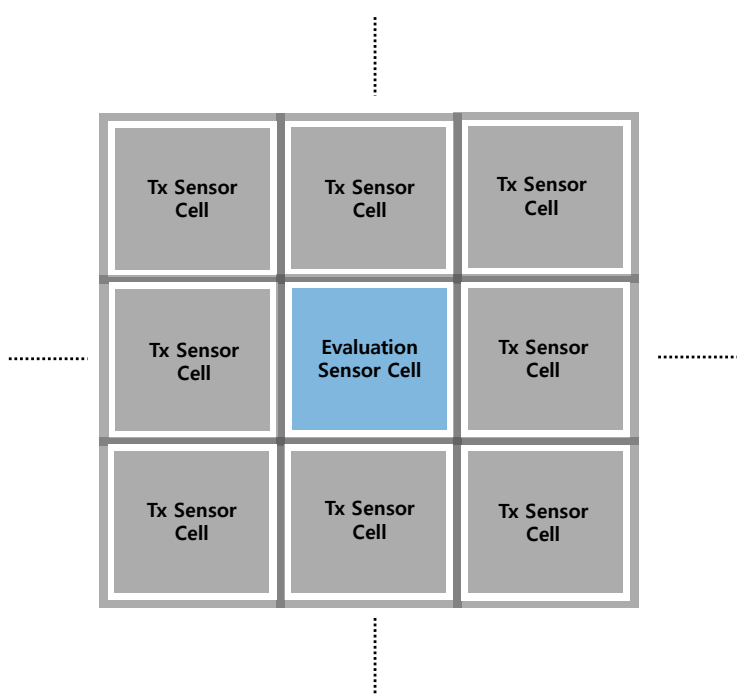

(a)

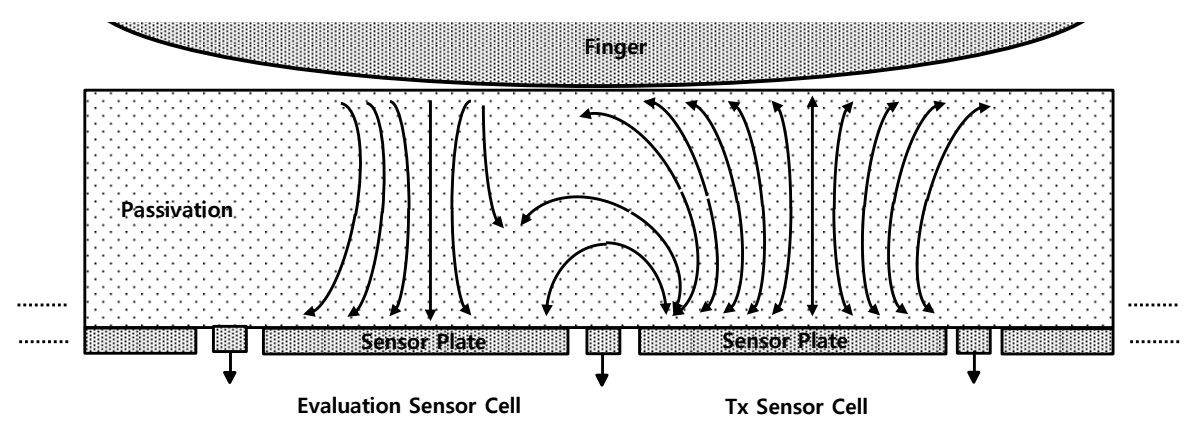

(b)

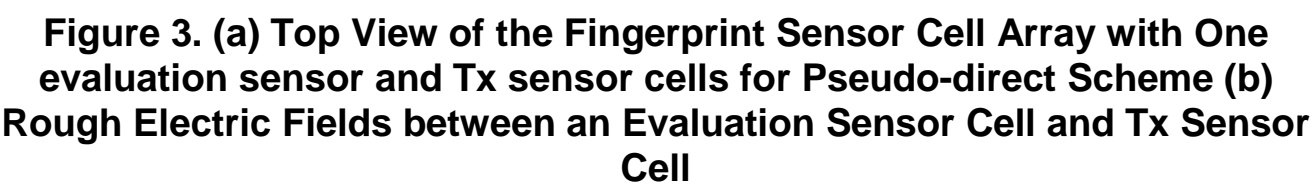

Figure 4 shows the top layout view of part of the proposed fingerprint sensor cell array for pseudo-direct scheme that includes an evaluation sensor cell, Tx sensor cells, and isolation sensor cells. As shown in Figure 4(a), the center sensor cell is for evaluation and the adjacent eight sensor cells are for isolation and other cells are for Tx signal transmission. The isolation sensor cells separate the evaluation sensor cell from the adjacent Tx sensor cells so that the direct inflows of the electric fields from the adjacent Tx sensor cells into the evaluation sensor cell are suppressed by increasing the gap between the evaluation sensor cell and the Tx sensor cells. Consequently, the evaluation sensor cell is properly evaluated by blocking the inflows of the Tx electric fields effectively. Forming isolation sensor cells in double or more layers could decrease the direct inflows of electric fields from Tx sensor cells by increasing the gap between the evaluation cells and Tx sensor cells as shown in Figure 4(b). These can be called isolation shields. Obviously, any shape of shields can be acceptable if it block the direct inflows of electric fields from Tx electrodes. And it is also possible to evaluate several sensor cells by evaluating multiple cells simultaneously or a pipelined scan evaluations[6]. 


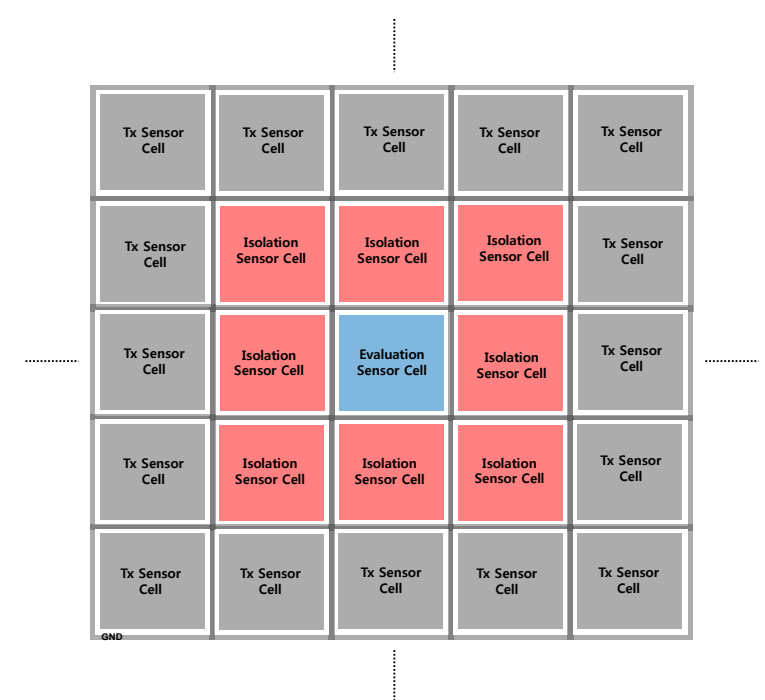

(a)

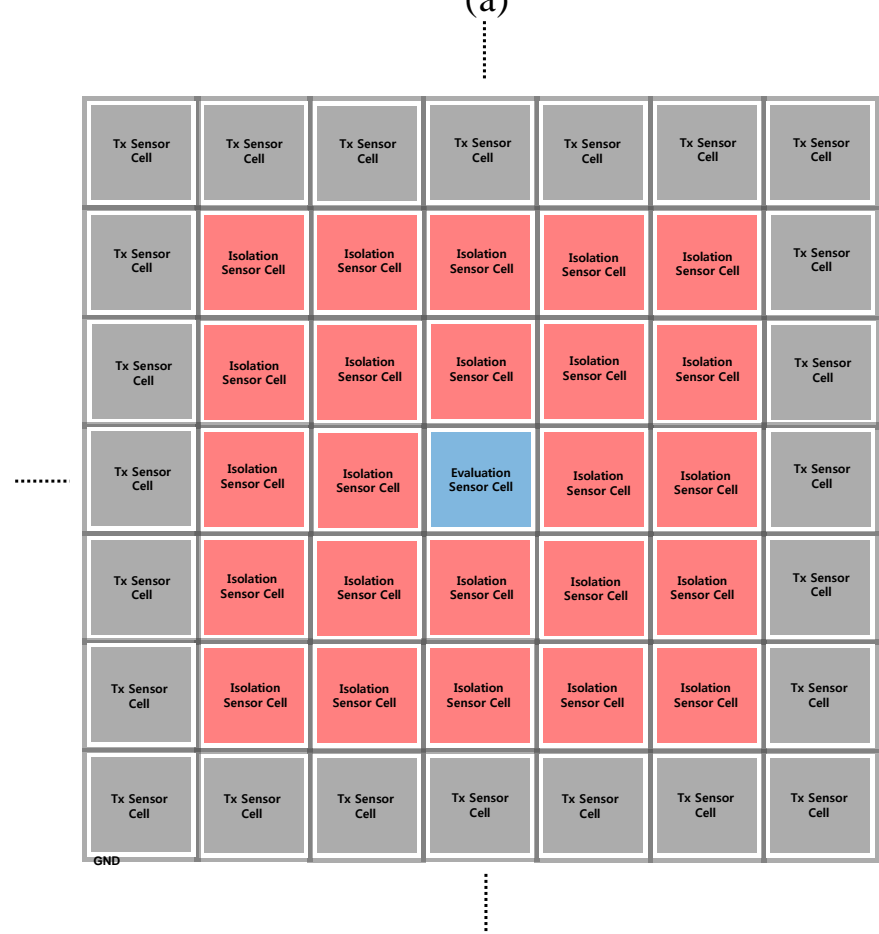

(b)

Figure 4. Top View of the Proposed Fingerprint Sensor Cell Array with One Evaluation Sensor and Tx Sensor Cells for Pseudo-direct Scheme with (a) one Layer of Isolation Sensor Cells, and (b) Two Layers of Isolation Sensor Cells

Figure 5 shows the rough electric fields for the sensor cells when an isolation sensor cell separates the evaluation sensor cell and Tx sensor cell. Most of the Tx electric fields toward a finger and the fringe electric fields are absorbed by the isolation sensor cell. Therefore, the evaluation sensor cell performs evaluation using the electric fields from a finger, which are originally from Tx sensor cells. If the passivation film becomes thicker, the more isolation sensor cell layers are required in order not to be affected because the electric fields from a finger to the evaluation sensor cell become weak while the direct inflows of electric fields remains almost the same. 


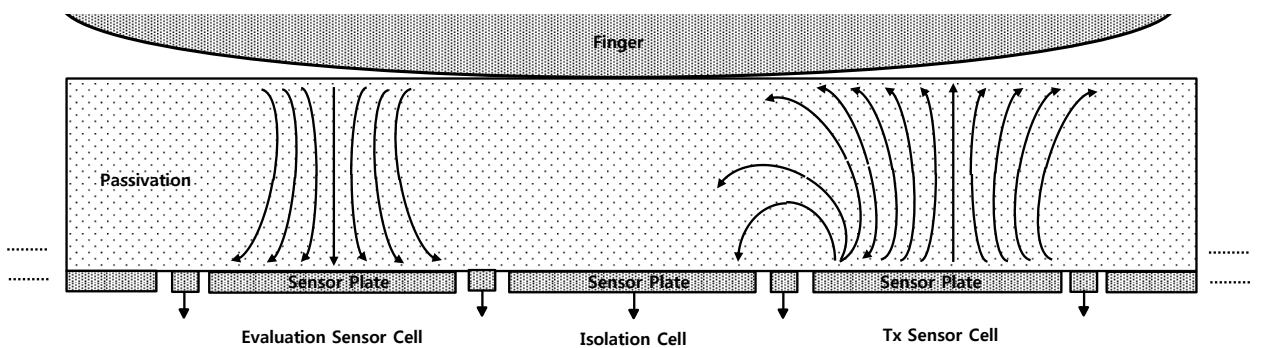

Figure 5. The Rough Electric Fields between One Evaluation Sensor Cell and Tx Sensor Cell with one Layer of Isolation Sensor Cell for the Proposed Fingerprint Sensor

Figure 5 shows the vertical layout view of the three adjacent fingerprint sensor cells of the proposed fingerprint sensor for pseudo-direct scheme. First, the evaluation sensor cell disables a NMOS switch for ground tie and a CMOS switch for Tx signal(SDRV) transmission so that the evaluation sensor cell operate normally for the proper evaluation. The integrator of the sensor cell is working for ridge and valley evaluation using two phase non-overlapping clocks. Second, the isolation sensor cell enables a NMOS switch to tie the sensor plate to the ground and disables a CMOS switch so that it blocks the electric fields from Tx sensor cells by separating the evaluation sensor cell and Tx sensor cell. Third, Tx sensor cell enables a CMOS switch to pass Tx signals to the sensor plate and disables a NMOS switch for ground tie. Lots of Tx sensor cells in the sensor array might form an good Tx electrodes with large capacitance.

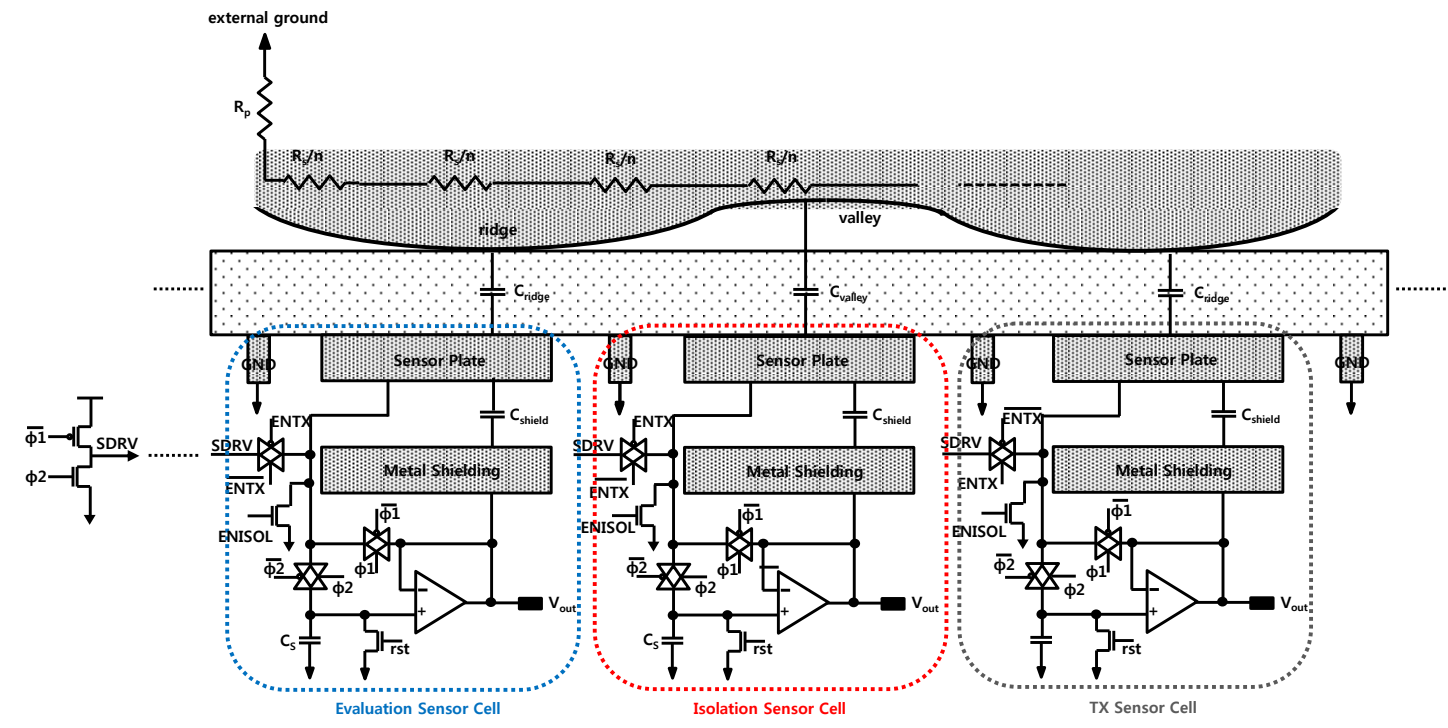

Figure 6. The Vertical Layout View of the Proposed Fingerprint Sensor Structure for Three Adjacent Fingerprint Sensor Cells, an Evaluation Sensor Cell, an Isolation Sensor Cell, and a Tx Sensor Cell

\section{Simulations}

The proposed fingerprint sensor cell with pseudo-direct scheme and a sensor cell isolation scheme has been simply modeled as shown Figure 2(a). If a 64x64 fingerprint sensor cell array is assumed, then the total fingerprint sensor cells are 4096. So, the number of Tx sensor cell which transmit Tx signal(SDRV) signal are more than 4000 for $64 x 64$ fingerprint sensor cell array. The Tx signal generated from a Tx signal driver using 
$10 \mathrm{MHz}$ two-phase non-overlapping clocks. If it is assumed a sensor plate of $50 \mu \mathrm{m} \times 50 \mu \mathrm{m}$ with a $100 \mu$ m-thick passivation film of permittivity of $4, \mathrm{C}_{\text {ridge }}$ is estimated as $1 \mathrm{fF}$. If the ridges and the valleys of a fingerprint are evenly distributed in the 64x64 fingerprint sensor cell array, then total capacitance of Tx electrodes which contact a ridges are roughly estimated about 2000. So, total capacitance of the Tx electrode can be estimated about $2 \mathrm{pF}$ which is sufficient to drive Tx signals to a finger for the proper sensor cell evaluation in the pseudo-direct scheme[*].

Figure 7 shows the output voltage characteristics of the proposed fingerprint sensor of pseudo-direct scheme with $\mathrm{C}_{\mathrm{Tx}}$ of $2 \mathrm{pF}, \mathrm{R}_{\mathrm{P}}$ of $1 \mathrm{M} \Omega, \mathrm{R}_{\mathrm{S}}$ of $500 \mathrm{k} \Omega$ and various $\mathrm{C}_{\text {gap }}$. $\mathrm{C}_{\text {gap }}$ varies with distance of isolation between an evaluation sensor cell and a Tx sensor cell. The output voltage after 15 -time charge integration $0.6 \mathrm{~V}, 0.87 \mathrm{~V}, 1.11 \mathrm{~V}$ for $\mathrm{C}_{\text {gap }}$ of $0 \mathrm{fF}$, $0.5 \mathrm{fF}$ and $1 \mathrm{fF}$, respectively. The output voltage with $\mathrm{C}_{\mathrm{gap}}$ of $1 \mathrm{fF}$ is almost doubled because $\mathrm{C}_{\mathrm{gap}}$ is added to $\mathrm{C}_{\text {finger }}$ for the charge integration process. The simulation results shows that $\mathrm{C}_{\text {gap }}$ directly affects the sensor's output voltage.

If $\mathrm{C}_{\text {gap }}$ is negligible compare to $\mathrm{C}_{\text {ridge }}, \mathrm{V}_{\text {sense }}$ will be much affected by $\mathrm{C}_{\text {gap }}$ not only by $\mathrm{C}_{\text {finger, }}$, where $\mathrm{V}_{\text {sense }}$ is defined as $\mathrm{V}_{\text {ridge }}-\mathrm{V}_{\text {valley. }}$. Note that $\mathrm{C}_{\text {finger }}$ decreases in proportion to the thickness of the passivation film. Therefore, $\mathrm{C}_{\text {gap }}$ should also be decreased by increasing the isolation distance so that the evaluation process would not be affected by the direct inflows of the electric fields from Tx electrodes.

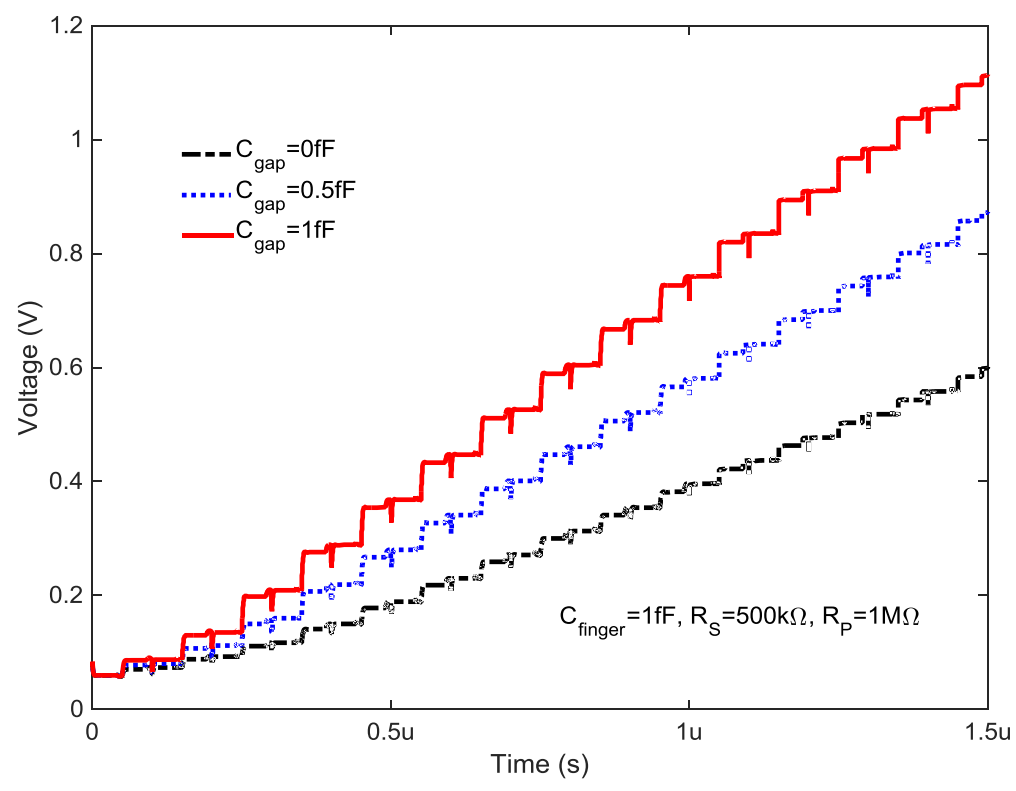

Figure 7. The Sensor's Output Voltage Characteristics with Various $\mathrm{C}_{\text {gap }}$ and Fixed $C_{\text {finger }}=1 \mathrm{fF}, R_{\mathrm{S}}=500 \mathrm{~K} \Omega$, and $R_{\mathrm{P}}=1 \mathrm{M} \Omega$

Figure 8 shows the output voltage characteristics during 15-time charge integrations of the proposed fingerprint sensor with $\mathrm{C}_{\mathrm{Tx}}$ of $2 \mathrm{pF}, \mathrm{R}_{\mathrm{P}}$ of $1 \mathrm{M} \Omega, \mathrm{R}_{\mathrm{S}}$ of $500 \mathrm{k} \Omega, \mathrm{C}_{\text {gap }}$ of $0.5 \mathrm{fF}$ and $1 \mathrm{fF}$. $\mathrm{C}_{\text {ridge }}$ of $1 \mathrm{fF}$ and $\mathrm{C}_{\text {valley }}$ of $0.2 \mathrm{fF}$ is assumed for the evaluation. $\mathrm{V}_{\text {sense }}$ after 15-time charge integration is $0,44 \mathrm{~V}, 0.4 \mathrm{~V}$ for $\mathrm{C}_{\text {gap }}$ of $0.5 \mathrm{Ff}, 01 \mathrm{fF}$ respectively. Obviously, since $\mathrm{V}_{\text {sense }}$ is affected by $\mathrm{C}_{\text {gap }}$ due to the additional charges injection through $\mathrm{C}_{\text {gap }}$, the output voltage saturates rapidly. 


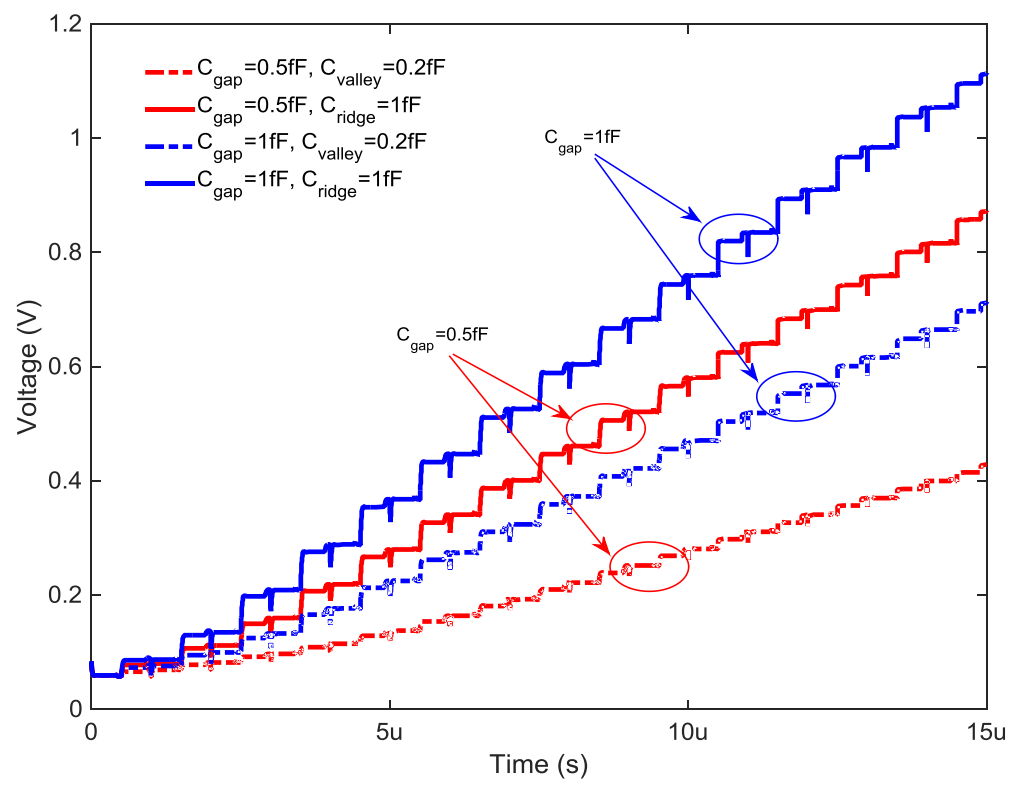

\section{Figure 8. The Sensor's Output Voltage Characteristics for $\mathrm{V}_{\text {sense }}$ Evaluation with $C_{\text {gap }}$ of $0.5 \mathrm{fF}, 1 \mathrm{fFand} \mathrm{C}_{\text {ridge }}=1 \mathrm{fF}, \mathrm{C}_{\mathrm{valley}}=0.5 \mathrm{fF}, \mathrm{R}_{\mathrm{S}}=500 \mathrm{~K} \Omega$, and $R_{P}=1 M \Omega$.}

Figure 9 shows $\mathrm{V}_{\text {sense }}$ with five $\mathrm{C}_{\text {gap }}$ after 15-time charge integrations. The simulation conditions were the same as in the previous simulation. $\mathrm{V}_{\text {sense }}$ is estimated as about $0.5 \mathrm{~V}$ and $0.25 \mathrm{~V}$ for $\mathrm{C}_{\text {gap }}$ of $0 \mathrm{fF}$ and $2 \mathrm{fF}$, respectively. $\mathrm{V}_{\text {sense }}$ decreases as $\mathrm{C}_{\text {gap }}$ increases because the direct charge injections come into the sensor cell through $\mathrm{C}_{\text {gap }}$. From the simulation results, we can see that $\mathrm{V}_{\text {sense }}$ is roughly inversely proportional to $\mathrm{C}_{\text {gap }}$. If the output voltage begins to saturate to VDD, then $\mathrm{V}_{\text {sense }}$ decreases rapidly.

Figure 10 shows the output voltage characteristics of the proposed fingerprint sensor with $\mathrm{R}_{\mathrm{P}}$ of $200 \mathrm{~K} \Omega, 600 \mathrm{~K} \Omega$, and $1 \mathrm{M} \Omega$ and fixed $\mathrm{C}_{\mathrm{Tx}}=2 \mathrm{pF}, \mathrm{R}_{\mathrm{S}}=500 \mathrm{~K} \Omega, \mathrm{C}_{\mathrm{gap}}=0.5 \mathrm{fF}$. $\mathrm{R}_{\mathrm{P}}$ is a modeled shunt resistor formed between a finger and external ground. The resistance of $R_{P}$ is usually in the range of between hundreds kilo to mega ohms. $R_{S}$ is a modeled series resistor of a finger. The resistance of $R_{S}$ can be predicted to have a smaller value than that of $\mathrm{R}_{\mathrm{P}}$ because the distance between the evaluation sensor cells and the Tx sensor cells in contact with a finger is usually shorter than that of a finger to external ground. In the simulation, the worst case $R_{P}$ and $R_{S}$ are assumed in order to estimate the external ground effect on the sensor cell evaluation.

As shown in Figure 10, the output voltage characteristics is not greatly affected by $R_{P}$ for the fingerprint sensor using an AOVF integrator. The output voltage characteristics is not much affected by $R_{P}$ as shown in Figure 10. Consequently, it can be predicted the proposed fingerprint sensor has a good performance even in mobile devices made of metal case with a ground connection. 


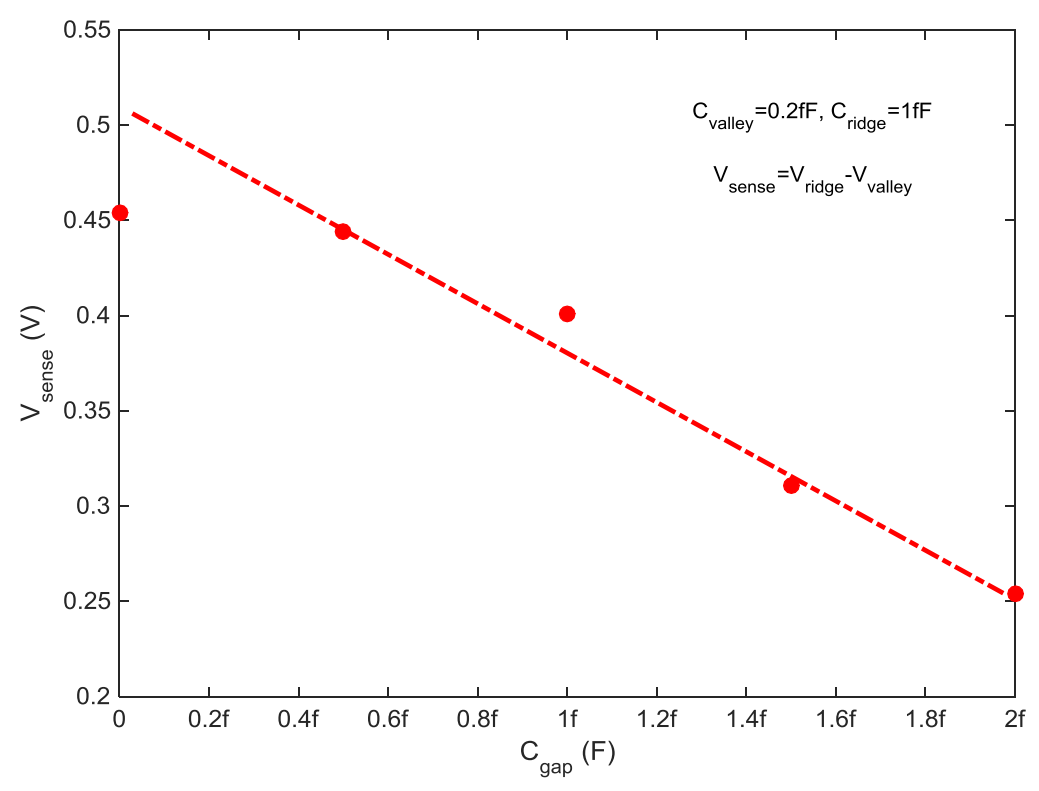

Figure 9. 3-dimensional Normalized Transfer Characteristics of the Circuit Model of Combined a Fingerprint and Tx Electrode of the Proposed Pseudo-direct Fingerprint Sensor with $\mathbf{C}_{\text {peri }}$ and $\mathbf{R}_{\mathbf{P}}$.

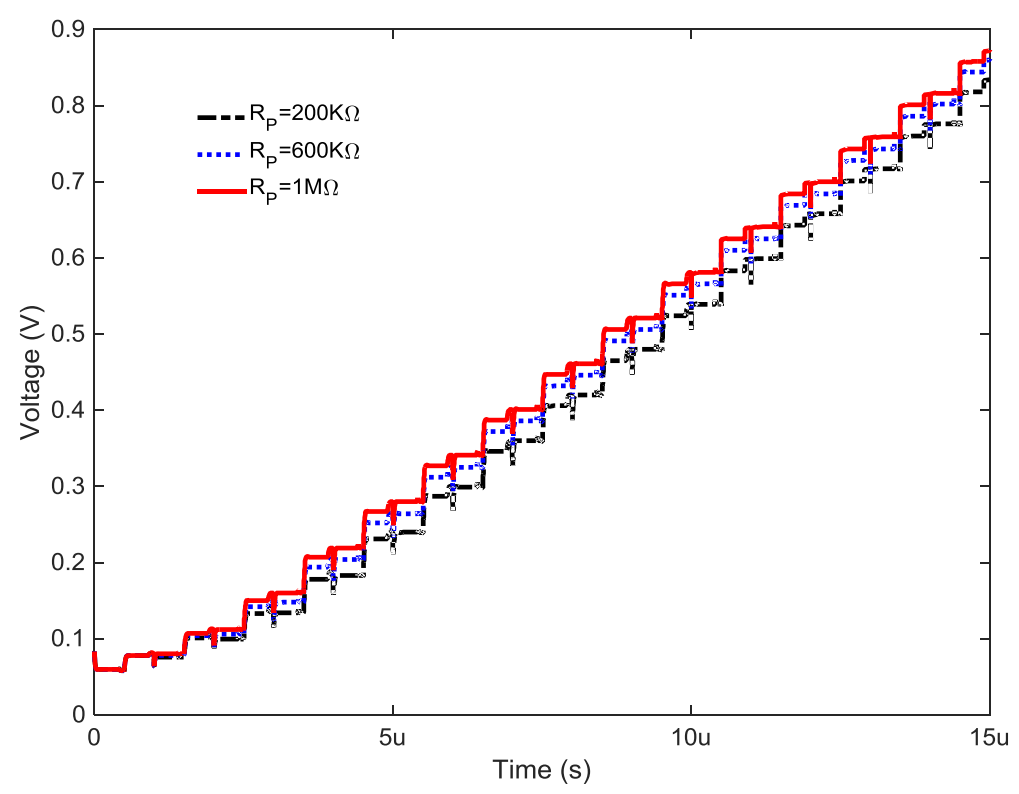

Figure 10. (a) Output Voltage with $C_{\text {finger }}$ of $1 \mathrm{fF}$ and (b) Vsense Characteristics for 20-time Signal Integrations Versus $\mathbf{R}_{\mathbf{P}}$

\section{Conclusion}

This paper proposed a fingerprint sensor cell structure and multiple use of a sensor cell that are an evaluation sensor cell, an isolation sensor cell, and a Tx sensor cell. The evaluation sensor cell senses fingerprint by sensing $\mathrm{C}_{\text {finger }}$. The isolation sensor cell isolates the evaluation sensor cell and the Tx sensor cell. The Tx sensor cell drive a Tx signal to a finger. Tx electrodes of sufficient capacitance 
can be formed by using a large number of Tx sensor cells. The isolation scheme of the evaluation sensor cells from the Tx sensor cells that improves the performances of the sensor cell evaluation by blocking the direct inflows of the electric fields generated from the adjacent Tx sensor cells was also proposed in this paper. The simulation verified that the proposed fingerprint sensor improves the performance of the fingerprint sensor with pseudo-direct scheme by increasing the capacitance of the Tx electrodes. And the simulation also verified that the shunt resistance formed between a finger and an external ground does not much affect on the sensor cell evaluation of the proposed fingerprint sensor.

Consequently, the proposed fingerprint sensor cell structure and the isolation schemes of the evaluation sensor cell can effectively be used in a fingerprint sensor of pseudo-direct scheme without an external electrode. Moreover, it can reduce a chip size of a fingerprint sensor by eliminating surrounded Tx electrode of the pseudo-direct fingerprint sensor proposed in [5].

\section{Acknowledgements}

This work was supported by Hanshin University Research Grant.

\section{References}

[1] D. R. Setlak, and G. Gozzini, Electronic Device Including Device Ground Coupled Finger Coupling Electrode and Array Shielding Electrode and Related Methods, U.S. Patent US2013/0314105, Nov. (2013)

[2] G. S. Franza, G. T. Minteer, J. G. Dooly, P. J. Landy, D. R. Setlak, and G. Gozzini, Finger Biometric Sensing Device Including Error Compensation Circuitry and Related Methods, U.S. Patent US2013/0315451, Nov. (2013).

[3] D. R. Setlak, N. W. V. Vonno, R. Lowther, and D. Gebauer, Electric Field Fingerprint Sensor Having Enhanced Feature and Related Methods, U.S. Patent 5940526, Aug. (1999)

[4] S. P. Hotelling, J. M. Bussat, and B. B. Lyon, Capacitive Sensing Array Modulation, U.S. Patent US20130271422, Oct. (2013).

[5] H. Yeo, "Capacitive Fingerprint Sensor Circuit using Pseudo-Direct Scheme for Mobile Applications," International Journal of Multimedia and Ubiquitous Engineering, (2015), Vol. 10, No. 2, pp237-248.

[6] H. Yeo, "A Fingerprint Sensor with Pipelined Scan Driver for Mobile Applications," International Journal of Multimedia and Ubiquitous Engineering, (2016), Vol. 11, No. 8, pp359-366..

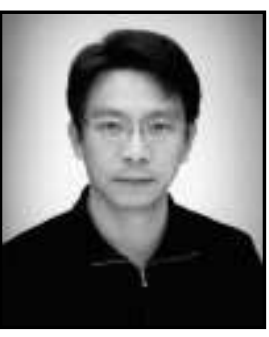

\section{Authors}

Hyeopgoo Yeo, He received his B.S. and M.S. degrees in electronic engineering from Yonsei University in Seoul, South Korea, in 1991 and 1993, respectively. He also received his M.S. and Ph.D. degrees in electrical and computer engineering from the University of Florida in Gainesville, FL, USA, in 2003 and 2007, respectively. From 1993 to 1999, he worked as a design engineer at Samsung Electronics Co., Ltd, where he performed ASIC cell library and highspeed digital I/O design using various CMOS technologies for gatearray and standard cell. In 2008, Dr. Yeo joined the hardware R\&D group at Samsung, where he was involved with mobile hardware design for wireless communications. In March 2009, he joined Hanshin University and he is currently an Assistant Professor. His research interests include high-speed serial link systems, data communication systems, and RF/analog circuit. 
International Journal of Control and Automation

Vol. 10, No. 6 (2017) 